\title{
Reproducción de la vida, superexplotación y organización popular en clave feminista: Una lectura desde Argentina
}

\author{
Reproduction of life, superexploitation, and popular organization from a feminist \\ perspective: A reading from Argentina
}

\author{
Juliana Agustina Díaz Lozano \\ diazlozano.juliana@gmail.com \\ Centro de Investigaciones Geográficas / Instituto \\ de Investigaciones en Humanidades y Ciencias \\ Sociales (CIG-IdIHCS) del Consejo Nacional de \\ Investigaciones Científicas y Técnicas (CONICET) - \\ Universidad Nacional de La Plata (UNLP), Argentina

\section{Mariano Féliz \\ marianfeliz@gmail.com \\ Centro de Investigaciones Geográficas / Instituto de \\ Investigaciones en Humanidades y Ciencias Sociales \\ (CIG-IdIHCS) del Consejo Nacional de \\ Investigaciones Científicas y Técnicas (CONICET) - \\ Universidad Nacional de La Plata (UNLP), Argentina}

Recepción: 02 Marzo 2020

Aprobación: 01 Junio 2020

Publicación: 02 Agosto 2020

Cita sugerida: Díaz Lozano, J. A. y Mariano, F. (2020). Reproducción de la vida, superexplotación y organización popular en clave feminista: Una lectura desde Argentina. Cuestiones de Sociología, 23, e101. https://doi.org/10.24215/23468904e101
Resumen: Este trabajo se pregunta sobre cómo se articula el patriarcado del salario con el proceso de superexplotación de la fuerza de trabajo (SEFT). La categoría SEFT, acuñada por Mauro Marini, opera como atractor en torno al cual se articula el proceso de valorización del capital en la economía dependiente. El trabajo reproductivo ocupa un lugar clave en garantizar condiciones materiales y simbólicas adecuadas para la producción continua de un flujo de fuerza de trabajo disponible para ser directamente explotado por el capital. En el capitalismo, el patriarcado del salario es un medio fundamental para la expropiación del trabajo de las mujeres. ¿Puede servir el concepto de superexplotación del trabajo de reproducción y cuidados para entender la dinámica específica de las economías dependientes? Desarrollaremos una discusión en torno a la naturaleza del concepto de superexplotación y su vinculación con el proceso de explotación del trabajo de las mujeres en economías dependientes.

Palabras clave: Superexplotación, Trabajo reproductivo, Patriarcado del salario, Economías dependientes, Organización popular.

Abstract: In this paper, we discuss the articulation of the patriarchy of wage with the process of superexploitaion of labor. This category, developed by Mauro Marini, operates to articulate the process of valorization of capital in dependent economies. The reproductive labor occupies a key place in guaranteeing material and symbolic conditions for the continuous production of labor force available to be directly exploited by capital. In capitalism, the patriarchy of wage is a key means for the expropriation of women's labor. Can the concept of superexploitation of labor in reproduction and care work be useful for understanding the specific dynamics of dependent economies? We will develop a discussion around the nature of this concept and its ties with the process of exploitation of women's labor in these economies.

Keywords: Superexploitation, Reproduction work, Patriarchy of wage, Dependent economies, Popular organization. 


\section{INTRODUCCIÓN}

El análisis de las condiciones de vida y -también- de los márgenes habilitados para la resistencia de los sectores populares en los países dependientes se enriquece con la articulación de enfoques críticos. En este sentido, los hallazgos de la economía feminista y de la teoría feminista en general refrescan la mirada de los procesos de superexplotación del trabajo en nuestros entornos, evidenciando su carácter multidimensional e interseccional. Efectivamente, las relaciones de dominación y explotación a escala global no son neutras en términos de género, sino que se conforman de forma históricamente estructuradas sobre relaciones patriarcales de larga data. Esta imbricación de relaciones de clase, género y raza ha moldeado las lógicas que subyacen a la superexplotación del trabajo, por ejemplo, en nuestro continente, generando condiciones y experiencias de vida diferenciales para las personas según su género. A su vez, las respuestas organizadas resistentes a los mecanismos de superexplotación son, también, generizadas, y en nuestros entornos dependientes se corresponden con formas de visibilización de los trabajos reproductivos y procesos de colectivización creativos, encabezados por mujeres y feminidades populares.

La articulación de las dos miradas mencionadas, es decir, los estudios sobre las economías dependientes con los que abordan el llamado patriarcado del salario (Federici, 2018) habilita interesantes interrogantes. ¿Podemos hablar de la superexplotación de la fuerza de trabajo en la reproducción y cuidados como fundante al fin de la reproducción de la dependencia? ¿Qué tácticas generan las feminidades populares para lidiar con esta superexplotación? ¿Qué potencialidades y limitaciones inaugura la colectivización de la sostenibilidad de la vida y la creación de espacios comunes para lidiar con la superexplotación? Estas preguntas guían el presente trabajo, que apunta a abrir discusiones y plantear reflexiones preliminares para comprender y transformar las economías dependientes como las latinoamericanas.

\section{SUPEREXPLOTACIÓN DE LA FUERZA DE TRABAJO Y CAPITALISMO DEPENDIENTE}

Según Marini, la superexplotación de la fuerza de trabajo (SEFT) consiste en

reducir el consumo del obrero más allá de su límite normal, por lo cual 'el fondo necesario de consumo del obrero se convierte de hecho, dentro de ciertos límites, en un fondo de acumulacion de capital', lo cual implica un modo específico de aumentar el tiempo de trabajo excedente (2015, pp. 124-125; cursiva en el original).

Osorio Urbina (2018, p. 165) completa señalando que a partir de la superexplotación "se les impide a los obreros reproducirse en condiciones normales, afectando a su vez la reproducción normal de los hijos, que crecerán y se desarrollarán física y espiritualmente con las huellas del desfalco y la depredación".

En tal sentido, la SEFT opera como mecanismo que articula el proceso de valorización del capital en la economía dependiente. Según Marini (2015, p. 132) la SEFT es la esencia de la dependencia. Al respecto, Osorio Urbina (2018) señala que

en tanto fundamento del capitalismo dependiente, el sistema mundial es el punto de partida para el análisis de la superexplotación, allí en donde se integran y articulan las diversas economías y sus modalidades de explotación, y en donde el capital funciona como capital mundial. Osorio Urbina (2018, p. 166)

En ese estadío, el sistema mundial se conforma como unidad diferenciada de diversas formas de capitalismo, interdependientes. Por otra parte, explica que

en el capitalismo dependiente existen condiciones objetivas para que la superexplotación se constituya en un mecanismo fundamental de explotación por parte del capital (Osorio, 2018, p. 169).

Es importante señalar que la superexplotación no es sinónimo de pobreza absoluta o bajos salarios, como sugieren, por ejemplo, Cueva (1974) y Katz (2017). Al contrario, como señalan Féliz y Haro (2019), la misma 
remite a las condiciones de producción de valor, no simplemente a las condiciones de vida (Bambirra, 1978), y supone la transformación de parte del fondo de consumo obrero en fondo de acumulación. Los salarios pueden crecer durante un período de tiempo específico, pero si simultáneamente aumenta el valor de la fuerza de trabajo la superexplotación puede persistir. La prevalencia de superexplotación "niega [al trabajador o la trabajadora] las condiciones necesarias para reponer el desgaste de su fuerza de trabajo" (Marini, 2015; corchetes nuestros) pero estas condiciones son relativas a las situaciones materiales de producción en contextos históricos y geográficos específicos.

\section{LA CARA OCULTA DE LA SUPEREXPLOTACIÓN}

En años recientes, se ha abierto un debate intenso en torno al carácter de la superexplotación de la fuerza de trabajo y su papel para garantizar la continuidad de las relaciones capitalistas a escala global (Katz, 2017; Katz, 2018; Osorio, 2018). Sin embargo, ha quedado relegado en el debate la articulación que hay entre la SEFT definida en términos clásicos y la explotación (o superexplotación) del trabajo de reproducción y cuidados. En el marco de una revolución en los debates en ese campo, desde el feminismo y en particular desde la economía feminista, es clave problematizar la cuestión a los fines de enriquecer la teoría marxista de la dependencia.

La reproducción de la fuerza de trabajo asume de manera invisibilizada al trabajo de reproducción y cuidados (Dalla Costa, 2009). Ese trabajo reproductivo ocupa un lugar clave en garantizar condiciones materiales y simbólicas adecuadas para la producción continua de un flujo de fuerza de trabajo disponible para ser directamente explotado por el capital. Sin ese trabajo, la producción de plusvalía se torna en efecto imposible (Dalla Costa, 2009; Dalla Costa y James, 1972). Esto implica que la superexplotación de la fuerza de trabajo debe ser entendida como un proceso en un sentido ampliado, es decir que va más allá del mero espacio de producción directa de valor en los procesos de producción de mercancías. La superexplotación de la fuerza de trabajo en el sentido de Marini pone en el centro la idea de que la remuneración de la misma está por debajo de su valor. Sin embargo, esa interpretación parcializa el proceso de producción de la fuerza de trabajo en tanto mercancía.

Al invisibilizar el trabajo de reproducción y cuidados, esencial para producir la fuerza de trabajo, la categoría de superexplotación pierde fuerza explicativa. En efecto, esta situación cumple un doble rol. Por una parte, permite crear las condiciones para que el mismo sea impago o peor pago que el trabajo dit productivo. $\mathrm{Al}$ ignorarlo en su importancia social y ocultarlo al interior de las familias y comunidades, lo desvaloriza (Federici, 2011). Por otro lado, la construcción de trabajo de reproducción y cuidados como "improductivo" y oculto es paralela a la construcción de una fracción social de menor valía: las mujeres y personas feminizadas, quienes son garantes últimas de este trabajo por la ética reaccionaria de los cuidados (Pérez Orozco, 2014).

El capitalismo no es necesariamente identificable con el trabajo formal y asalariado, sino que es en esencia trabajo no libre (Federici, 2011). De esta manera, poner al trabajo reproductivo en el centro de la explicación del sistema capitalista permite comprender que este descansa sobre la producción de un tipo particular de trabajador, de familia y sexualidad, cuya reproducción involucra mucho más que el mero consumo de mercancías. De allí que sea clave la conexión umbilical entre la devaluación del trabajo reproductivo y la devaluación de la posición social de las mujeres (Federici, 2013, p. 161) y, por otra parte, que sea esencial para comprender la naturaleza profunda de la dependencia.

Este proceso de invisibilización es parte fundamental del orden del patriarcado. El mismo es un sistema que se asienta sobre la explotación del trabajo de las mujeres y su dominación económica, pero también sobre la explotación sexual, la heterosexualidad obligatoria y la quita de libertades y posibilidades de decisión sobre la vida propia. El patriarcado se constituyó -y se constituye todos los días- como uno de los basamentos de la reproducción del sistema capitalista (Rubin, 1996; Cháneton, 2007). Sin embargo, el patriarcado y el capitalismo se retroalimentan (Hartmann, 1980) llegando a articularse en una forma particular de patriarcado: el patriarcado del salario. Esto significa que el salario se constituye en un mecanismo esencial para 
el control y subordinación del trabajo de las mujeres por parte de los varones (Federici, 2018). En contextos dependientes, el patriarcado del salario configura un proceso de superexplotación del trabajo invisibilizado de las mujeres, proceso que es parte integral de la reproducción de la condición dependiente.

La base material sobre la que se asienta este sistema está en la expropiación del trabajo femenino bajo la forma de una discriminación abierta o encubierta de las mujeres (Federici, 2018), a quienes se excluye del acceso a bienes (o trabajo remunerado) y restringe su sexualidad. El matrimonio monogámico y heterosexual es la forma más reciente y eficaz de lograr ambas formas de exclusión. En este marco, la dominación masculina se ejerce no sólo contra las mujeres, sino contra todo sujeto feminizado que no cumpla con el estereotipo de varón, burgués, occidental, blanco, adulto, heterosexual y propietario (Haraway, 2004; Lorde, 2003). La forma de familia burguesa es el verdadero pilar oculto de la organización capitalista del trabajo pues resuelve el trabajo de cuidados o de reproducción de forma gratuita para el capital y para los varones.

Ahora bien, ¿cómo se organiza el patriarcado del salario en economías dependientes? ¿Podemos hablar de la superexplotación de la fuerza de trabajo en la reproducción y cuidados como fundante al fin de la reproducción de la dependencia?

\section{REPRODUCCIÓN DE LA VIDA Y SUPEREXPLOTACIÓN DEL TRABAJO DE CUIDADOS}

En el marco del patriarcado del salario, en los territorios dependientes la superexplotación del trabajo atraviesa todo el espacio de la reproducción social. Por ello, entre las mujeres trabajadoras y de sectores populares la superexplotación laboral no sólo se expresa en un proceso ligado al ciclo inmediato de valorización del capital (en formas de trabajo remunerado) sino que fundamentalmente es la carga del trabajo invisible de cuidados que se ve incrementada en los espacios de valorización dependientes.

Esta situación involucra en particular el volumen de trabajo requerido para la resolución de lo que Pérez Orozco (2014) denomina "desesidades" en el hogar: no sólo es necesario garantizar el sostén biológico de les integrantes del mismo sino fundamentalmente su sostén emocional. Esto se ve agravado en situaciones de crisis en el ciclo de reproducción capitalista:

Las mujeres son útiles no sólo porque llevan a cabo el trabajo doméstico sin salario y sin ir a la huelga, sino también porque acogen en la casa a todos los que periódicamente son expulsados de sus trabajos en las crisis económicas (Dalla Costa y James, 1972, p. 9).

En otras palabras, las mujeres sostienen la vida pero también sostienen el capital con sus cuerpos y sus múltiples trabajos. De manera general, la sobrecarga de trabajo sobre las mentes y cuerpos femeninos entremezcla labores remuneradas y no remuneradas, realizadas individual y colectivamente para sostener sus vidas y las de sus familias.

Esta multiplicidad de tareas recae especialmente sobre el tiempo y el cuerpo de las feminidades en hogares de sectores populares y suponen un trabajo físico y mental que no cesa. La cotidianeidad femenina supone un gran número de tareas entrelazadas y preocupaciones mezcladas en tiempo y lugar. En el análisis de las experiencias vitales de sobreexplotación femenina no se identifican momentos y espacios delimitados, es decir, trabajo remunerado fuera del hogar/trabajo no remunerado dentro. Al contrario, a partir de un uso intensivo del tiempo, las mujeres trabajadoras y de sectores populares corren de un lado al otro para resolver de forma contingente estas desesidades familiares, con horarios flexibles y tareas superpuestas.

En América Latina se estima que en alrededor de un tercio de los hogares las mujeres son sostén de hogar con responsabilidad central en el sustento material y emocional de la familia (Marchionni, Gasparini y Edo, 2019). Un informe de la CEPAL (2020) - previo a la crisis sanitaria del COVID-19- indica que en los países de la región sobre los que se dispone de datos las mujeres destinaban a las actividades de trabajo doméstico y de cuidados entre 22 y 42 horas semanales. 
En particular, cabe señalar que la reducción de las posibilidades de planificación y decisión sobre la maternidad (en especial, producto de legislación que penaliza el aborto) y los recortes en las políticas de salud sexual y reproductiva, coarta la autonomía de las mujeres pobres e influye en la ligazón femenina a la resolución de cuidados:

la prohibición de la autonomía a elegir y la restricción de la posibilidad voluntaria y legal en condiciones de garantía de salud, calidad y no penalización refuerza el hecho de maternidades obligadas que garantizan la reproducción social a muy bajo costo (Costantino y Laterra, 2018, p. 169).

De conjunto, esto implica que la superexplotación del trabajo de las mujeres se extiende con intensidad sobre el trabajo reproductivo no remunerado, ampliando de esa forma la apropiación capitalista (y patriarcal) de tiempo de trabajo a los fines de su reproducción ampliada. En contextos dependientes, el trabajo no remunerado de las mujeres posibilita la continuidad de la acumulación por parte del capitalismo -operando como parte del mecanismo de compensación del intercambio desigual (Marini, 2015)- pero también sostiene y contiene el desarrollo de la vida de las personas en un entorno crecientemente hostil, alienado y violento. Según la OIT (2016), las mujeres de los países dependientes dedican a la resolución del cuidado no remunerado casi dos horas diarias más que aquellas mujeres que viven en los países desarrollados. La superexplotación del trabajo de reproducción se traduce entonces en una sobrecompensación que el capital en territorios dependientes recibe con un enorme costo para las mujeres: su tiempo libre se reduce por encima de la media, la intensidad de su trabajo no pago se amplía, y si consiguen trabajo remunerado lo hacen también -en general- en condiciones de superexplotación clásica.

El trabajo no remunerado de las mujeres tiene en contextos dependientes dos sentidos: posibilita la continuidad de la acumulación por parte del capitalismo, pero también sostiene y contiene el desarrollo de la vida de las personas en un entorno crecientemente hostil. Desde esta perspectiva, los hogares actuarían comoámbitos donde se tramita el descontento y desorden social resultante, por ejemplo, del desempleo. Pero, al mismo tiempo, es en los hogares y barrios populares donde se sustentan las "desesidades" de las grandes mayorías.

Cabe aclarar que las mujeres y las disidencias sexuales no son meros objetos pasivos de esta relación, sino que históricamente se han revelado, por una parte, a la invisibilización y desvalorización del ámbito reproductivo y, por otra, a su confinamiento en esa esfera. Los Paros Internacionales de Mujeres, Lesbianas, Travestis y Trans de los 8 de Marzo de 2017, 2018 y 2019 bajo la consigna "Si nosotras paramos, se para el mundo" expresan estas resistencias a la superexplotación y al maltrato, abriendo el ámbito reproductivo a nuevas posibilidades de contestación y cambio social.

\section{TERRITORIOS DEPENDIENTES: REPRODUCCIÓN DE LA VIDA EN RIESGO}

En tiempos de crisis civilizatoria del capital como el actual (Mészáros, 2008), en los territorios dependientes operan formas renovadas de saqueo o desposesión que se combinan con nuevas formas de explotación del trabajo, bajo modalidades informales, ilegales y serviles (Gago y Mezzadra, 2015). Como parte de los nuevos saqueos y avances sobre el territorio (Cielo y Vega, 2015), se debilitan y destruyen modos de vida comunitarios que persisten, a través del despojo a comunidades de sus tierras y sus modos de subsistencia, buscando incorporarlos más eficazmente a la lógica de valorización del valor. Este proceso de reorganización global capitalista se constituye en un ataque sistemático sobre la reproducción de la vida y en particular contra las mujeres que tienen un papel principal en las disputas por el control de los territorios y los medios de subsistencia.

Por otra parte, y simultáneamente, en las fronteras del capital -allí donde se produce con virulencia una ofensiva capitalista patriarcal sobre la vida, reconfigurando, no sin resistencias, su organización precedente 
(Mezzadra y Neilson, 2017, p. 22)- el ataque sobre los territorios fuerza un incremento en los procesos de migración al interior del continente.

Amplios sectores de los pueblos afectados por el avance del saqueo se trasladan a través de las fronteras nacionales, para insertarse en los mercados laborales de los países más desarrollados. Dentro de los territorios dependientes, como América del Sur, mujeres de sectores populares son parte de un proceso de migración transnacional para incorporarse trabajando en oficios vinculados a los cuidados como niñeras, criadas, auxiliares de salud (Magliano, 2015). Las migrantes se insertan en las llamadas "cadenas globales de cuidados", es decir, redes transnacionales que se establecen para sostener cotidianamente la vida y a través de las cuales las mujeres se transfieren cuidados con base en ejes de jerarquización social (Pérez Orozco, 2014; Falquet, 2014). De esta manera, la crisis de los cuidados en los países centrales se conjuga con la crisis de la reproducción en países del sur, lo que resulta en el empleo a bajo costo de las mujeres migrantes para la resolución de los cuidados. Sin estas cadenas globales no sería posible la reproducción social -en su forma actual- en los países más desarrollados (Costantino y Laterra, 2018). En el mismo sentido, la crisis de reproducción en territorios dependientes expresa un acentuado proceso de superexplotación de la fuerza de trabajo femenina.

Volviendo a Argentina, según los datos de la Encuesta Permanente de Hogares (EPH), el trabajo doméstico remunerado representa un 16,7 \% de las asalariadas para el año 2014 y se encuentra fuertemente precarizado, ya que $78 \%$ de estas trabajadoras no están registradas en la seguridad social. Además, es una actividad fuertemente racializada, con un $25 \%$ de migrantes de países sudamericanos dentro del total de las trabajadoras de cuidados. Estos empleos socialmente desvalorizados, informales y mal pagados con frecuencia son la única opción para muchas mujeres migrantes, lo que manifiesta la jerarquización y segmentación étnico-nacional y de género del mercado de trabajo (Magliano, 2015) y la extensión de modos de precarización laboral a escala transnacional. Aquí podemos ver que los procesos de superexplotación del trabajo en la esfera de la reproducción también operan entre centros de acumulación capitalista dependientes con distintos grados de desarrollo capitalista. Las relaciones desiguales que se establecen entre territorios dependientes se producen tanto entre esferas productivas como en el espacio del trabajo de reproducción social: poblaciones migrantes son superexplotadas en territorios dependientes que se encuentran arriba en la estructura jerárquica de la dependencia. En el caso de Argentina, por ejemplo, mujeres migrantes de países lindantes desarrollan trabajos de reproducción social siendo superexplotadas y aportando al proceso de reproducción social y de valorización en el capitalismo dependiente del país. De esta forma, el mecanismo de compensación que se expresa en la forma superexplotación, encuentra un nuevo clivaje en los núcleos más desarrollados dentro del espacio dependiente.

\section{Políticas estatales y SUPEREXPLOTACión DE LA FUERZA DE TRABAJO DE LAS MUJERES}

El proceso de superexplotación de la fuerza de trabajo en territorios dependientes se produce a través de mecanismos diversos. En particular, juega un papel fundamental el accionar del Estado que arbitra medios para propender a la reproducción ampliada del capital. Las políticas sociales constituyen instrumentos privilegiados para la reproducción de condiciones de superexplotación en el trabajo reproductivo y de cuidados.

En las últimas décadas se produce una transición en la forma del Estado capitalista dependiente (Féliz, 2017). Esa transición supone, entre otras cosas, la configuración de una nueva generación de políticas sociales de asistencia básica hacia los sectores populares (Brown, 2020). En los albores del siglo XXI, en diversos países de la región suramericana se consolidan políticas de corte asistencialista promovidas por el Banco Mundial (Fiszbein y Schady, 2009). Para el año 2011 existían en América Latina unos 40 Programas de Transferencia de Ingresos Condicionada (TIC), que operaban en 18 países de la región y alcanzaban a más de 25 millones de hogares y a cerca de 113 millones de personas, lo que constituía el 19\% de la población de la región (Cecchini y Madariaga, 2011). Algunos de estos programas son la Asignación Universal por Hijo (AUH) en Argentina 
(casi 4 millones de niñes ${ }^{1}$ ), Bolsa Familia de Brasil ( 52 millones de personas), Oportunidades de México (27 millones de personas) y Familias en Acción de Colombia (12 millones de personas).

Estos programas sociales, que suponen en su mayoría transferencias monetarias condicionadas e individuales (Brown, 2020), son una forma de paliar la situación de vulnerabilidad de los sectores más empobrecidos, según el modelo del workfare (empleo forzoso) impulsado por los organismos internacionales de crédito (Svampa, 2009). Este proceso implica la financiarización de los derechos sociales.

Estas políticas constituyen mecanismos extractivistas (Gago y Mezzadra, 2015). A través de ellos, se expanden las fronteras del capital hacia espacios y sujetos construidos como marginales y periféricos, incluso a partir de la generación de nuevos sujetos de endeudamiento. En Argentina, la consolidación del programa AUH, creado en 2009, supone la monetarización del acceso a derechos básicos como la alimentación, la escolaridad y la salud de les niñes. Este programa estuvo acompañado con una política de créditos de consumo, otorgados por la Administración Nacional de la Seguridad Social (ANSES) a les beneficiaries del mismo. El sensible incremento del endeudamiento en este segmento afecta casi de manera exclusiva a las mujeres, que representan el $97 \%$ de quienes la perciben como titulares (ANSES, 2018).

Asentadas en la idea de que las personas actúan como agentes económicos racionales que buscan optimizar su situación, estas políticas tienden a reforzar el precepto que asigna a las personas pobres la responsabilidad individual sobre su situación de pobreza y en sus acciones (agencia) la posibilidad de superarla (Rodríguez Enríquez, 2011). Estos programas, que constituyen paliativos de las consecuencias sociales de los ajustes (garantizando un ingreso monetario mínimo masivo para las familias y colaborando con la reducción de los índices de indigencia), son parte de la estrategia de contención del conflicto social que se combina con mecanismos de represión y judicialización de la protesta y pobreza (Féliz y Pérez, 2007; Anzorena, 2013b). Estos programas TIC parten de un enfoque de corresponsabilidad que en realidad implica que el Estado asigne dinero a niñes con necesidades insatisfechas y les dé la responsabilidad de su resolución a las mujeres. De esta forma, aprovechan y refuerzan los roles genéricos tradicionales y el trabajo no remunerado de las mujeres, sin imponer condiciones para que los varones se involucren en las tareas de cuidados y sin proponer mecanismos y dispositivos estatales que aborden el reparto desigual de esta carga. Como afirma Schild (2015), las mujeres pasan a ser reclutadas para combatir la pobreza, en nombre de una idea fabricada de "empoderamiento", término que significaba convertir a las mujeres pobres en ciudadanas "más responsables" y "capaces" de sostener la vida familiar².

Por otra parte, se combinan con programas sociales de capacitación para el trabajo o de trabajo cooperativo. En Argentina el programa "Argentina Trabaja", devenido en "Hacemos Futuro" en la actualidad, ${ }^{3}$ está orientado mayormente al trabajo de mantenimiento o limpieza del espacio público; en la práctica se constituye como una forma de creación de trabajo precario (y superexplotado) por parte del Estado. En estas modalidades de contratación y explotación de la fuerza laboral a través de formas cooperativas (constituidas para poder acceder al programa), el Estado se desdibuja en tanto empleador y busca evitar las obligaciones formales vinculadas al empleo formal en el sector público, como los aportes jubilatorios, vacaciones pagas, Aseguradoras de Riesgos del Trabajo y demás cargas sociales.

En Argentina, la AUH se combinó con la apertura dentro del programa "Argentina Trabaja” de una línea específica que tiene como destinatarias a las mujeres de sectores populares: "Ellas Hacen” lanzada en 2013. A pesar de su objetivo explícito de trabajar con mujeres vulnerables y deconstruir roles de género en el trabajo, el programa no considera en su implementación las altas cargas de trabajo de estas mujeres en su vida cotidiana, por lo que termina sobrecargándolas con nuevas tareas relacionadas con la participación en el programa, ampliando así las condiciones para la superexplotación de su fuerza de trabajo no remunerada (Isola Zorrozúa, 2015).

En Argentina, estos programas producen una segmentación por género en las políticas sociales y de empleo: las principales políticas laborales se orientan a los varones (porción mayoritaria de la fuerza de trabajo empleada formalmente) desde el Ministerio de Trabajo, Empleo y Seguridad Social, y las políticas sociales se 
destinan fundamentalmente a las mujeres, ${ }^{4}$ impartidas desde el Ministerio de Desarrollo Social (Féliz y Díaz Lozano, 2018). De hecho, detrás de ellas persiste el estereotipado modelo de familia nuclear y heterosexual sobre el que se piensan las políticas sociales de redistribución (Anzorena, 2013b).

Esta nueva generación de políticas sociales propendieron a articular una nueva "economía de los cuidados" sobre las espaldas de las mujeres (Rodríguez Enríquez, 2005). Su eje articulador son las tareas de producción de aquellos valores de uso necesarios para la reproducción cotidiana de las personas. De esta forma, se produce una ambivalencia estatal (Anzorena, 2013). Con una retórica de ampliación de derechos para las mujeres en tanto ciudadanas, estas políticas no reconocen las problemáticas de género en vínculo con las determinaciones de clase social y raza. De esa forma, los programas no favorecen la redistribución de la riqueza hacia la base de la pirámide social: las mujeres pobres y, en algunos casos, migrantes. Esta ambivalencia da cuenta de la ausencia de un cuestionamiento a la división social y sexual del trabajo, la feminización de la pobreza y los mecanismos de segregación racial que reproducen la estructura social. Es decir, desde el punto de vista de la articulación del trabajo productivo y reproductivo, en términos generales, estas políticas operaron de manera de reproducir los roles tradicionalmente asignados a mujeres y varones, reproduciendo valores del cuidado como destino natural y asentado esencialmente sobre el esfuerzo de las mujeres.

Paradójicamente, estas políticas sociales destinadas a fortalecer el rol de las mujeres como cuidadoras se instalan en el continente al tiempo que las luchas feministas se masifican, proponiendo, entre otras cosas, otra división por género del trabajo y conmoviendo la organización social del cuidado a escala global. Conviven incluso con cambios normativos positivos en términos del reconocimiento identitario y derechos ciudadanos como la legislación sobre salud sexual y reproductiva, contra la violencia de género, en favor de la educación sexual, etc. Estas iniciativas de reconocimiento tienen como objetivo "remediar la injusticia cultural" (Fraser, 1997) y coexisten contradictoriamente durante todo el período con políticas de redistribución, es decir, aquellas que pretenden "reparar la injusticia económica" (Fraser, 1997, p. 25), pero que en la práctica no logran conmover o afectar las desigualdades.

\section{REPRODUCCIÓN DE LA VIDA, ORGANIZACIÓN POPULAR Y SUPEREXPLOTACIÓN}

¿Cómo se vivencian estos momentos de despojo y ajuste estructural en los sectores populares, y fundamentalmente desde las mujeres que constituyen el sostén de la reproducción de las familias y comunidades?

En general, como dijimos, con un reforzamiento y sobrecarga de trabajo sobre sus cuerpos y en usos intensivos del tiempo. Es decir, para las mujeres de sectores populares la sobrecarga de trabajo es una situación permanente, porque ellas acceden a los trabajos más flexibles y precarios (situación no alterada por las políticas sociales de las cuales son destinatarias privilegiadas) pero también porque, a pesar de trabajar parte del tiempo fuera de la casa, el trabajo doméstico y de cuidados sigue recayendo sobre sus cuerpos (Féliz y Díaz Lozano, 2018). En los entramados comunitarios, esta dinámica intrahogares se traslada en parte- a las tareas de reproducción de la vida en los barrios y en las organizaciones, donde las mujeres garantizan mayoritariamente la alimentación, vestimenta, cuidados infantiles, arreglo de infraestructura y mantenimiento barrial, asistencia a reuniones organizativas, entre otras actividades diarias.

Desde las teorías feministas, y específicamente desde la economía feminista, se ha utilizado la idea de doble jornada para ilustrar esta sobrecarga de trabajo. A pesar de la utilidad de este concepto para visualizar la desigualdad de género (Rodríguez Enríquez, 2015), la idea de múltiples presencias en relación a lo laboral resulta más pertinente en el caso de las mujeres de sectores populares con trabajos informales (Sciortino, 2018).

Pensar las dinámicas de doble y hasta triple presencia en trabajo asalariado, reproductivo y comunitario, describe una vinculación de las mujeres con el trabajo en la actualidad caracterizada por la acumulación y mezcla en espacio y tiempo de trabajos con lógicas distintas. Las múltiples presencias femeninas se 
visualizan en la tarea cotidiana de combinar de manera flexible el trabajo en el mercado, asalariado, con las actividades reproductivas domésticas, muchas veces ni siquiera visibilizadas socialmente como trabajo. Pensar esta situación desde la idea de presencias, permite relativizar las dicotomías que estructuran el trabajo en el capitalismo fordista: presencia/ausencia, público/privado, trabajo/no trabajo, productivo/reproductivo (Carrasquer Oto, 2009). Permite, además, entender cómo la mayor flexibilidad en los tiempos y espacios de trabajo en economías dependientes se traduce en un mayor esfuerzo por parte de las mujeres para poder organizar y gestionar la vida cotidiana. Esto se profundiza y potencia en las experiencias de las mujeres de sectores populares debido a las situaciones de precariedad vital (Díaz Lozano, 2020).

Como contracara, esta sobrecarga de trabajo puede generar un fortalecimiento de los lazos de interdependencia entre las personas, sobre todo a escala territorial y comunitaria. Con interdependencia nos referimos a las relaciones que se establecen -en este caso en los barrios populares- a partir del conjunto de de actividades y trabajos interconectados en común para garantizar la reproducción simbólica, afectiva y material de la vida (Echeverría, 1998; Gutiérrez Aguilar, Navarro Trujillo y Linsalata, 2017; Navarro Trujillo, 2018). Así, las tramas de interdependencia protagonizadas por mujeres van autorregulándose y desplegando su capacidad para darse forma colectivamente y gestionar algunos aspectos de la vida social. Desde esta perspectiva, se hace hincapié en el aspecto relacional del trabajo de cuidados, es decir que es una actividad centrada en el reconocimiento de la vulnerabilidad humana y la interdependencia, por lo que la defensa de la vida está en el centro. Los cuidados intentan llegar donde el Estado no garantiza y el consumo no llega, a esos espacios dañados por la lógica de acumulación (Pérez Orozco, 2014).

La necesidad de sustentar la vida, entonces, de resolver "desesidades" que el Estado desatiende y que no pueden suplirse adquiriendo bienes y servicios en el mercado, comienza a pasar cada vez más por la posibilidad del "rebusque" (Mendoza, 2015) individual y también colectivo: desde las redes familiares de cuidados, donde fundamentalmente las mujeres organizan precariamente horarios y trabajos dentro de los hogares -pero también en las redes establecidas entre mujeres de diferentes hogares-, hasta el establecimiento de nuevas relaciones y espacios comunitarios o el fortalecimiento de los ya existentes.

Evidentemente, como correlato del saqueo y el ajuste, las tácticas individuales de rebusque se combinan con la instauración o fortalecimiento de instancias colectivas, como plantean Gutiérrez Aguilar, Navarro Trujillo y Linsalata (2017), con procesos inéditos de autorganización social, o en otros casos de la actualización de prácticas sociales preexistentes de producción de comunes y ampliación de la gestión comunitaria hacia ámbitos que anteriormente no estaban regulados de esa manera. Vemos surgir o crecer en nuestras regiones, en estos momentos apremiantes, diversos espacios de autogestión comunitaria: los llamados comedores populares, roperos comunitarios, copas de leche, bachilleratos populares, clubes del trueque y ferias americanas, espacios de cuidados infantiles - mal llamadas "guarderías"-, a veces con algún aporte estatal, pero generalmente como iniciativa autoorganizada desde los territorios populares. Esta extensión de los cuidados a lo comunitario genera lo que Verónica Gago y Lucy Cavallero (2020) denominan la única infraestructura insustituible, en prácticas y dispositivos que resuelven cuidados ante "lo que ha sido sucesivamente privatizado, despojado, desfinanciado" (2020, p. 1).

Por no estar organizadas estas experiencias y relaciones en función del lucro, sino de la sostenibilidad de la vida, pueden posibilitar el surgimiento de lógicas de organización distintas, asentadas en la centralidad de la cooperación, la deliberación colectiva, la equidad, e incluso la propiedad colectiva. Es decir, estas experiencias de lo común que ponen en el centro la sostenibilidad de la vida son pasibles de escenificar la capacidad de las personas de dar forma, de autodeterminar fines, ritmos y formas de su vida práctica (Gutiérrez Aguilar, Navarro Trujillo y Linsalata, 2017; Linsalata, 2015), lo que estes autores nombran como formas comunitarias de lo político, es decir, "dinámicas asociativas o lógicas de reproducción de la vida colectiva cuya reiteración en distintos contextos y momentos de la vida social nos permiten distinguir con claridad los rasgos de una forma peculiar de la política” (Linsalata, 2015, p. 16). 
Esto se evidencia, por ejemplo, en la pluralidad de formas organizativas que sustentan estos comunes, las prácticas de democracia directa, de trabajo cooperativo y sin patrón, la rotación de responsabilidades, entre otras que se establecen en los barrios populares (Díaz Lozano, 2018). Es cada vez más clara la centralidad femenina en estos comunes, debido a que se sostienen en gran medida a partir de la colectivización de actividades de cuidados que comúnmente realizan las mujeres. Por ejemplo, en parte de la bibliografía sobre el movimiento piquetero en Argentina se muestra cómo, ante la creciente desocupación a fines de la década de los años 90, fueron fundamentalmente las mujeres las que se organizaron en espacios comunitarios y de protesta, dando sustento cotidiano y territorial a estas organizaciones (Andújar, 2014; Causa, 2007; Partenio, 2008; Cross y Freytes Frey, 2007; Díaz Lozano, 2018 entre otras).

\section{DiSPUTA DE LO COMUNITARIO: ENTRE EL SOSTENIMIENTO DE LA VIDA Y LA ASIMILACIÓN}

Si consideramos la idea de "doble movimiento" de Polanyi (2009), podemos decir que, contra el avance permanente de la lógica de acumulación del capital sobre la vida de las personas, lo comunitario es parte de un movimiento de autodefensa de la sociedad, de una apuesta de la vida contra la lógica del despojo (Caffentzis y Federici, 2015). En otras palabras, un movimiento de reapropiación de riquezas y capacidades sociales por parte de las personas "de a pie". Las iniciativas de las mujeres de sectores populares han desempeñado un papel especial en este contexto, por su ligazón más débil con el mercado laboral formal y por su histórica responsabilidad en los trabajos reproductivos.

En nuestros territorios dependientes los espacios y vínculos comunitarios se crean y se actualizan en el marco de un sistema hostil para toda lógica que no esté organizada desde la ganancia. Es decir, estamos en presencia de una pragmática vitalista neoliberal (Gago, 2015) que combina tanto prácticas de superexplotación y financiarización individual, como formas comunitarias que están disputando palmo a palmo con esas lógicas, pero que también pueden ser condicionadas, apropiadas, redefinidas por este poder.

Para citar un ejemplo de cómo el sistema puede disputar e incluso intentar utilizar estos vínculos y espacios comunitarios, retomamos un artículo publicado en el conservador diario La Nación de Buenos Aires. En él se presenta un elogio a la práctica de los comedores barriales como una forma de "cambio de vida" para "enfrentar a la crisis". Es decir, en momentos de un ajuste que no se cuestiona, desde los sectores hegemónicos ponderan estas prácticas autoorganizadas por los sectores populares como parte de la solución para disminuir los impactos de las medidas económicas en aquellos. En consonancia, desde los sectores concentrados se ofrece, como salida a la crisis, el apoyo estatal a estas experiencias como forma de paliar sus consecuencias a bajo costo, y a su vez, contener los posibles efectos del descontento social. En el mismo sentido, se elogia el desempeño de las mujeres en estas experiencias desde su rol maternal y de responsables de los cuidados. Caffentzis y Federici (2015) advierten sobre el uso del capital y del Estado de lo comunitario, como intentos de cooptación de estas experiencias porque proveen formas de reproducción a bajo costo, o -si se quiereporque constituyen formas de superexplotación de la fuerza de trabajo de las mujeres en el trabajo de reproducción.

Como se mencionó con anterioridad, las políticas de impulso estatal al trabajo cooperativo también constituyen en la práctica formas de utilización de las lógicas solidarias y comunitarias; en este caso, para la legalización del trabajo precario. Estas políticas que integran mayoritariamente a mujeres bajo la figura de cooperativas de trabajo constituyen, en los hechos, precarización laboral que, en su mayor parte, ocupa mujeres de sectores populares. Pueden asimismo reforzar lazos clientelares institucionalizando, en muchos casos, la asignación de roles y tareas de cuidados a las mujeres (Anzorena, 2013; Féliz y Díaz Lozano, 2018) al no proponer o fomentar modos de funcionamiento cooperativos en términos prácticos en la toma de decisiones o la propiedad colectiva. El uso de la figura de cooperativa para la organización de muchos de estos programas institucionaliza el fraude laboral por parte del Estado. Y estas mismas políticas han mantenido la evolución en el valor monetario de los beneficios de las diferentes modalidades de cooperativas de trabajo, 
subsidios para trabajo comunitario y programas de transferencia condicionada, las cuales están muy por debajo de la evolución general de las remuneraciones de los asalariados formalizados. ${ }^{5}$

Asimismo, estas políticas - a medio camino entre el empleo y el desempleo, entre la inclusión y la exclusión social- son incompatibles con otras actividades registradas para las mujeres, lo cual en la práctica puede reforzar su sobrecarga de trabajo y las múltiples presencias laborales.

Claro está que, a pesar de las condiciones impuestas por las políticas, se han relevado interesantes estrategias individuales y colectivas por parte de las mujeres y las organizaciones populares para colectivizar, hacer comunitario y cuestionar la distribución de dichos trabajos para así otorgar significados alternativos a estos ingresos y suscitar prácticas autogestivas colectivas, utilizando como base el ingreso de las políticas sociales que originalmente fueron planteadas como de beneficio individual (Sciortino, 2018, Díaz Lozano, 2018).

Advertir el peligro de que los comunes sean absorbidos por la lógica del capital, o funcionalizados, no implica desconocer su potencial para crear nuevos vínculos surgidos desde el movimiento de reapropiación colectiva de las capacidades de reproducción de la vida. En el marco de un sistema que avanza en la totalización de las relaciones dominadas por la relación de valor, lo comunitario puede contribuir a instalar formas de "destotalización" (Linsalata, 2015), negando la lógica predominante. El Estado y el capital nunca pueden subsumir o enajenar por completo estas experiencias comunitarias de destotalización, porque las prácticas comunes están presentes como construcción histórica en el pueblo, que las reactualiza en el presente.

Cuando están enfocadas desde lógicas de búsqueda de autonomía política y económica, las experiencias comunitarias pueden habilitar espacios para pensar otras formas sociales, otros modos de "resolver la vida". Por eso, es necesario preguntarse por las formas políticas que se disputan en lo comunitario, los mecanismos de deliberación, los modos de gestión de las necesidades, las modalidades de jerarquía que se instalan, y de las potencialidades prefigurativas de estas relaciones, que ponen el foco en el resguardo de la vida y no la ganancia. Es aquí donde estas formas comunitarias pueden vincularse con las perspectivas feministas anticoloniales que buscan construir entramados políticos no estructurados desde la lógica de la dominación masculina, occidental, burguesa, que separa dirigentes y dirigides, quienes piensan y quienes hacen, quienes sobresalen en lo público y quienes realizan el trabajo invisible en lo privado. Justamente, Raquel Gutiérrez Aguilar (2015) define como "política en femenino" a estas formas políticas inauguradas desde lo común que no escinden la práctica cotidiana de sostenimiento de los debates "políticos".

Crear y sostener comunes es una práctica que forma parte de los recursos populares. En economías dependientes los comunes buscan resolver las vidas precarizadas, colectivizar necesidades, oponerse al saqueo permanente. Sin embargo, lo comunitario no tiene un contenido contrahegemónico per se, sino que, como toda relación social, está atravesado por una pugna permanente por su definición. Advertirlo así es central para pensar las articulaciones entre las experiencias y evadir los intentos permanentes de ruptura, asimilación o morigeración impulsadas desde el sistema.

Es interesante rescatar el gran volumen de experiencia femenina en la gestión de estos comunes, y las estrategias de reorganización y colectivización del trabajo de reproducción de la vida que históricamente han realizado. A partir de estas múltiples experiencias, las mujeres han pugnado por romper el aislamiento hogareño del trabajo doméstico, han creado formas de lucha desde estos cuidados colectivizados, y han revisado los lugares femeninos tradicionales. Estas luchas de las mujeres contra el confinamiento al trabajo en el hogar es una parte de la historia de combate al capitalismo patriarcal que apenas se está comenzando a rescatar y reconstruir. El espesor político de la colectivización de los cuidados tiene que ver con la ruptura de dicotomías patriarcales y modernas: lo personal/lo político y lo público/lo privado. Los límites interiores a estas supuestas antinomias se vuelven difusos cuando surgen espacios comunes para la gestión de la vida. Primero porque le da una nueva visibilidad, y luego porque genera una posibilidad de encuentro y organización de las mujeres a partir de lógicas de cooperación antagónicas con la individualización del capital (Díaz Lozano, 2018). Por consiguiente, lo comunitario enfocado desde un punto de vista feminista puede inaugurar un lugar resistente desde donde disputar al avance del capital sobre la vida. Es, de alguna manera, 
una forma de enfrentar la tendencia general del capitalismo dependiente a ampliar la superexplotación de la fuerza de trabajo al espacio de la reproducción y el cuidado.

\section{REFLEXIONES FINALES}

La superexplotación de la fuerza de trabajo es el fundamento de la dependencia. Esta abarca los espacios de producción directa de plusvalía hasta las esferas de reproducción y cuidados. El trabajo reproductivo invisibilizado y desvalorizado- ocupa un lugar clave en las economías dependientes para garantizar condiciones materiales y simbólicas para la producción continua de un flujo de fuerza de trabajo disponible para el capital. Sin ese trabajo, la producción de plusvalía se torna en efecto imposible.

En nuestros territorios, la superexplotación del trabajo atraviesa el espacio de la reproducción social, incrementándose la carga del trabajo de cuidados en los espacios de valorización dependientes. Por consiguiente, la sobrecarga de trabajo sobre las mentes y cuerpos femeninos entremezcla tareas remuneradas y no remuneradas, configurando un trabajo físico y mental que no cesa, con tareas superpuestas que impiden pensar en términos de jornadas laborales delimitadas. El patriarcado del salario opera en estos territorios dependientes como mecanismo fundante de formas diversas de superexplotación de la fuerza de trabajo de las mujeres en el espacio de la reproducción.

Las feminidades populares, que tienen en este patriarcado del salario la responsabilidad central del sustento material y emocional de las familias y comunidades, viven una precarización mayor de sus vidas y una superexplotación mayor de las diferentes formas de trabajo que realizan. La superexplotación del trabajo no remunerado amplía la apropiación capitalista y patriarcal del tiempo de trabajo a fines de la reproducción ampliada del capital.

En contexto de crisis, vemos en nuestras latitudes instaurarse formas renovadas de saqueo o desposesión que se combinan con nuevos modos de explotación del trabajo debilitándose modos de vida comunitarios que se van integrando a la lógica del valor. Esto conlleva un ataque sistemático a la reproducción de la vida y en particular contra las mujeres que tienen un papel principal en la defensa de territorios y modos de subsistencia. Paralelamente desde el Estado se mantienen políticas sociales que combinan mecanismos de financiarización de derechos con programas que hacen uso de los roles genéricos tradicionales para garantizar cuidados y contención a bajo costo.

La contracara de la superexplotación laboral y la sobrecarga femenina es el fortalecimiento de lazos de interdependencia comunitaria, espacios de autogestión, lógicas de organización distintas como parte de un movimiento de autodefensa de la sociedad contra el despojo. Estos intentos de destotalización y de reapropiación de riquezas y capacidades sociales entran en un diálogo tenso con las iniciativas del Estado que, al tiempo que ajusta y reprime a los sectores populares, intenta apropiarse de ciertas experiencias comunitarias para usarlas como paliativos sociales ante la crisis.

Fortalecer lo disruptivo de lo comunitario tiene que ver con fortalecer las lógicas de organización que no reproduzcan la dominación, que no se sometan al Estado o al mercado; que mantengan disputas en clave anticapitalista y antipatriarcal en su interior y de cara a la sociedad. En esto estriba precisamente la necesidad imperiosa de la articulación de los feminismos con estas experiencias de sostenibilidad de la vida en colectivo.

De esta manera, para las mujeres de los países dependientes la profundización de las tendencias actuales del capitalismo transnacional significa una precariedad mayor de sus vidas y una superexplotación de su trabajo. La superexplotación del trabajo en el espacio de la reproducción y los cuidados supone que la intensificación de esa forma de trabajo se multiplica de forma tal de no poder ser compensada por las posibilidades de reproducir la vida en condiciones medias. En buena parte de los hogares y comunidades, las mujeres se ven forzadas a buscar empleos en cualquier condición o autogenerarse formas de ingreso informales (Fraser, 1997). Por otro lado, frente a la desarticulación del Estado de bienestar, las mujeres pobres y trabajadoras deben reforzar su carga de trabajo reproductivo en los hogares y la comunidad. 
Nos preocupa, por lo tanto, cuando experiencias comunitarias presentes, por ejemplo, como motor de organizaciones populares emblemáticas como las campesinas, de Derechos Humanos, barriales, terminan siendo apropiadas y/o condicionadas por las lógicas partidarias o las propuestas de gobiernos. En esta relación siempre desigual, lo primero que se resiente es la lógica política comunitaria, esta "política en femenino" que había inventado una nueva forma de lidiar con la sostenibilidad de la vida. Los comunes anticapitalistas, por tanto, no sólo deben pensarse, como en el ejemplo de los comedores populares, como espacios colectivos de resolución de necesidades, sino esforzarse por renovar cotidianamente la apuesta por la autonomía política y, al mismo tiempo, la articulación con otras experiencias para fortalecerse y ser realmente una alternativa futura para la organización social.

Los comunes pueden ser fortalecidos por el cúmulo de nuevas prácticas y saberes generados al calor de los feminismos, en este momento de auge del movimiento. La lucha por la visibilización de los trabajos invisibles de reproducción de la vida, la necesidad de enfocarnos en la interdependencia humana y con la naturaleza, la denuncia del capitalismo patriarcal colonial como sistema de despojo de nuestros cuerpos y territorios y la posibilidad de formas políticas no opresivas son solo algunas de las apuestas feministas cada vez más difundidas.

\section{REFERENCIAS}

Andújar, A. (2014). Rutas argentinas hasta el fin. Mujeres, politica y piquetes 1996-2001. Buenos Aires: Ediciones Luxemburg.

ANSES (2018). Datos Abiertos Asignaciones Universales. Disponible en https://www.anses.gob.ar/informacion/dato s-abiertos-asignaciones-universales.

Anzorena, C. (2013). Mujeres en la trama del Estado. Una lectura feminista de las políticas públicas. Mendoza: Editorial de la Universidad Nacional de Cuyo (Ediunc).

Anzorena, C. (2013b). Desigualdades que persisten: el lugar de las mujeres en las políticas sociales (Argentina 2000 a 2010). Nómadas, Revista Crítica de Ciencias Sociales y Jurídicas, Núm. especial: América Latina. Disponible en h ttps://webs.ucm.es/info/nomadas/americalatina2013/claudiaanzorena.pdf.

Bambirra, V. (1978). Teoria de la dependencia: una anticritica. México: Ediciones Era.

Brown, B. (2020). Mercados de trabajo segmentados y politicas sociales. Un estudio sobre la (re)configuración de la matriz socio-asistencial en Argentina (2003-2015) (Tesis doctoral inédita). Universidad de Buenos Aires, Buenos Aires, Argentina.

Caffentzis, G. y Federici, S. (2015). Comunes contra y más allá del capitalismo. Revista Apantle, 1, 51-72.

Carrasquer Oto, P. (2009). La doble presencia. El trabajo y el empleo femenino en las sociedades contemporáneas (Tesis doctoral). Universidad Autónoma de Barcelona, Barcelona, España.

Causa, A. (2007). La configuración de la trama de mujeres desocupadas (piqueteras). La acción colectiva antes, durante y después de los piquetes. Actas del V Congreso Europeo de Latinoamericanistas, Bruselas.

Cecchini, S. y Madariaga, A. (2011). Programas de Transferencias Condicionadas. Balance de la experiencia en América Latina y el Caribe. Recuperado de CEPAL: https://repositorio.cepal.org/bitstream/handle/11362/27854/S20 11032_es.pdf?sequence $=1$ \&isAllowed $=\mathrm{y}$

CEPAL (2010). Programas de transferencias condicionadas benefician a más de 100 millones de personas en la región. Recuperado de https://www.cepal.org/es/comunicados/programas-transferencias-condicionadas-benefician-m as-100-millones-personas-la-region

CEPAL (2020). La pandemia del COVID-19 profundiza la crisis de los cuidados en América Latina y el Caribe. Recuperado de https://repositorio.cepal.org/bitstream/handle/11362/45335/5/S2000261_es.pdf.

Cháneton, J. (2007). Género, poder y discursos sociales. Buenos Aires: Eudeba.

Cielo, C. y Vega, C. (2015). Reproducción, mujeres y comunes. Nueva Sociedad, 256. Recuperado de http://nuso.org /articulo/reproduccion-mujeres-y-comunes-leer-a-silvia-federici-desde-el-ecuador-actual/ 
Costantino, A. y Laterra, P. (2018). La teoría marxista de la dependencia desde una mirada feminista: un análisis sobre las leyes sobre aborto en el mundo. Cuadernos CEMARX, 10.

Cross, C. y Freytes Frey, A. (2007). Movimientos piqueteros: tensiones de género en la definición del liderazgo. Argumentos, 20(55), 77-94. Recuperado de http://www.redalyc.org/articulo.oa?id=59505503

Cueva, A. (1974). Problemas y perspectivas de la teoría de la dependencia. Revista Historia y Sociedad, 3, 55-77.

Dalla Costa, M. y James, S. (1972). El poder de la mujer y la subversión de la comunidad. México: Siglo XXI.

Dalla Costa, M. (2009). Reproducción y migración. En M. Dalla Costa, Dinero, perlas y flores en la reproducción feminista (pp. 57-98). Madrid: Akal.

Díaz Lozano, J. (2018). Mujer bonita es la que sale a luchar. Experiencias de vida de mujeres participantes del Frente Popular Dario Santillán de Berisso (Tesis de doctorado inédita). Universidad Nacional de La Plata, Facultad de Humanidades y Ciencias de la Educación, La Plata, Argentina. Recuperado de http://www.memoria.fahce.unl p.edu.ar/tesis/te.1800/te.1800.pdf.

Díaz Lozano, J. (2020). Triple presencia femenina en torno a los trabajos: mujeres de sectores populares, participación política y sostenibilidad de la vida. Revista Tempo e Argumento, 12(29). Recuperado de http://www.revistas.ud esc.br/index.php/tempo/article/view/2175180312292020e0108

Echeverría, B. (1998). Valor de uso y utopia. México: Siglo XXI.

Falquet, J. (2014). Hacia un análisis feminista y dialectico de la globalización neoliberal: El peso del complejo militaroindustrial sobre las «mujeres globales». Revista Internacional de Pensamiento Politico, 9.

Federici, S. (2011). Calibán y la bruja. Mujeres, cuerpo y acumulación originaria. Buenos Aires: Tinta Limón Ediciones.

Federici, S. (2013). La reproducción de la fuerza de trabajo en la economía global y la inacabada revolución feminista. En F. Silvia, Revolución Punto Cero (pp. 153-180). Madrid: Traficantes de Sueños. Recuperado de https://www .traficantes.net/sites/default/files/pdfs/Revolucion\%20en\%20punto\%20cero-TdS.pdf.

Federici, S. (2018). El patriarcado del salario. Críticas feministas al marxismo. Madrid: Traficantes de Sueños.

Féliz, M. (2017). Acumulación de capital y lucha de clase(s) en y a través del Estado en la Argentina neodesarrollista. Theomai, 35, 171-186.

Féliz, M. Haro, A. (2019). Dependencia, valor y naturaleza. Hacia una revitalización crítica de la teoría marxista de la dependencia. Revista Sociedad, 38, 45-56.

Féliz, M. y Díaz Lozano, J. A. (2018). Trabajo, territorio y cuerpos en clave neodesarrollista. Argentina, 2002-2016. Revista Perfiles Latinoamericanos, 26(52), 1-26. DOI: https://10.18504/pl2652-005-2018

Féliz, M. y Pérez, P.E. (2007). ¿ ¿Tiempos de cambio? Contradicciones y conflictos en la política económica de la posconvertibilidad. En R. Boyer y J.C. Neffa (ed), Salidas de crisis y estrategias alternativas de desarrollo. La experiencia argentina (pp. 319-352). Buenos Aires: Editorial Miño y Dávila.

Fiszbein, A. y Schady, N. (2009). Transferencias monetarias condicionadas Reducción de la pobreza actual y futura. Washington: Banco Mundial. Recuperado de https://openknowledge.worldbank.org/bitstream/handle/1098 6/2597/476030PUB0SPAN1h0Transfers10Spanish.pdf?sequence=6\&isAllowed=y.

Fraser, N. (1997). ¿De la redistribución al reconocimiento? Dilemas en torno a la justicia en una época 'postsocialista'. En N. Fraser, Iustitia Interrupta: Reflexiones críticas desde la posición "postsocialista” (pp. 17-54). Bogotá: Siglo del Hombre Editores.

Gago, V. (2015). La razón neoliberal. Buenos Aires: Tinta Limón.

Gago, V. y Cavallero, L. (2020). Crack Up! Feminismo, pandemia y después. Recuperado de https://www.cadtm.org/ Crack-Up-Feminismo-pandemia-y-despues.

Gago, V. y Mezzadra, S. (2015). Para una crítica de las operaciones extractivas del capital Patrón de acumulación y luchas sociales en el tiempo de la financiarización. Revista Nueva Sociedad, 255. Recuperado de https://nuso.o rg/media/articles/downloads/4091_1.pdf

Gutiérrez Aguilar, R., Navarro Trujillo, M. y Linsalata, L. (2017). Repensar lo político, pensar lo común. Claves para la discusión. Recuperado de https://kutxikotxokotxikitxutik.files.wordpress.com/2017/11/pensar-lo-politico-p ensar-lo-comun_gutierrez-navarro-linasalata-clavescomunfinal.pdf. 
Gutiérrez Aguilar, R. (2015). Políticas en femenino. Transformaciones y subversiones no centradas en el Estado. Revista Contrapunto, 7, 123-139.

Haraway, D. (2004).Testigo_Modesto@Segundo_Milenio. Barcelona: Editorial UOC.

Hartmann, H. (1980). Un matrimonio mal avenido, hacia una unión más progresiva entre feminismo y marxismo. Zona Abierta, 24, 85-113.

Isola Zorrozúa, F. (2015). Las posibilidades de movilidad social en un grupo de mujeres, pobres y vulneradas (Área de San Vicente, Provincia de Buenos Aires, 2013-2015). Actas del III Seminario Internacional Desigualdad y Movilidad Social en América Latina, Bariloche, Argentina Recuperado de http://www.memoria.fahce.unlp.edu .ar/trab_eventos/ev.9379/ev.9379.pdf.

Katz, C. (2017). Aciertos y problemas de la superexplotación. Rebelion.org. Recuperado de http://www.rebelion.org /noticia.php?id=231417.

Katz, C. (2018). Controversias sobre la superexplotación. Vientosur.info. Recuperado de http://vientosur.info/spip. php?article13568.

Linsalata, L. (2015). Cuando manda la asamblea: lo comunitario-popular en Bolivia: una mirada desde los sistemas comunitarios de agua de Cochabamba. México: Sociedad Comunitaria de Estudios Estratégicos.

Lorde, A. (2003). La hermana, la extranjera. Articulos y conferencias. Madrid: Horas y Horas.

Magliano, M.J. (2015). Interseccionalidad y migraciones: potencialidades y desafíos. Revista Estudos Feministas, 23, 691-712.

Marchionni, M., Gasparini, L., y Edo, M. (2019). Brechas de género en América Latina. Un estado de situación. Caracas: CAF. Recuperado de http://scioteca.caf.com/handle/123456789/1401.

Marini, R.M. (2015). Dialéctica de la dependencia (1973). En C.E. Martins (ed.), América Latina, dependencia y globalización (pp. 107-50). Buenos Aires: Siglo XXI Editores / CLACSO.

Mendoza, P. (2015). Aproximaciones teóricas a la práctica del rebusque en Bogotá. Papeles de Coyuntura, 40.

Mészáros, I. (2008). The Challenge and Burden of Historical Time: Socialism in the Twenty-First Century. Nueva York: Monthly Review Press.

Mezzadra, S. y Nielson, B. (2017). La Frontera como método. Madrid: Traficantes de Sueños. Recuperado en https:// www.traficantes.net/sites/default/files/pdfs/PC15_frontera_como_metodo.pdf

Ministerio de Desarrollo Social (2015). Ier Informe: Antecedentes, creación y primera etapa del Programa Ellas Hacen. Recuperado en https://www.desarrollosocial.gob.ar/wp-content/uploads/2015/07/2.-1er-informe-antecedent es-creacion-y-1er-etapa.pdf.

Navarro Trujillo, M.L. (2018). Hacer común contra la fragmentación en la ciudad. Dinámicas de autonomía e interdependencia. En R. Gutiérrez A. (ed.), Debates contemporáneos sobre Comunalidad, lo comunitario y la producción de lo común. Oaxaca: Pez en el árbol.

OIT (2016). Las mujeres en el trabajo: Tendencias de 2016. Ginebra: OIT.

Osorio Urbina, J. (2018). Acerca de la superexplotación y el capitalismo dependiente. Cuadernos de Economía Critica, 4(8), 153-181.

Partenio, F. (2008). Género y participación politica: Los desafíos de la organización de las mujeres dentro de los movimientos piqueteros en Argentina. Programa Regional de Becas CLACSO. Recuperado en http://biblioteca virtual.clacso.org.ar/ar/libros/becas/2008/deuda/partenio.pdf

Pérez Orozco, A. (2014). Subversión feminista de la economia. Aportes para un debate sobre el conflicto capital-vida. Madrid: Traficantes de Sueños.

Polanyi, K. (2009). El sustento del hombre. Madrid: Capitán Swing.

Rodríguez Enríquez, C. (2005). Economía del cuidado y politica económica: una aproximación a sus interrelaciones. Trabajo presentado en la Trigésima octava reunión de la Mesa Directiva de la Conferencia Regional sobre la Mujer de América Latina. CEPAL, Mar del Plata.

Rodríguez Enríquez, C. (2015). El trabajo de cuidado no remunerado en Argentina: un análisis desde la evidencia del Módulo de Trabajo no Remunerado. Buenos Aires: Equipo Latinoamericano de Justicia y Género. 
Rodríguez Enríquez, C. (2011). Programas de transferencias condicionadas de ingreso e igualdad de género. ¿Por dónde anda América Latina?. Santiago de Chile: CEPAL.

Rubin, G. (1996). El tráfico de mujeres: Notas sobre la "economía política" del sexo. En M. Lamas (ed.). El género: la construcción cultural de la diferencia sexual (pp. 35-96). México: PUEG.

Sciortino, S. (2018). El trabajo de cuidado entre las titulares del programa social "Ellas Hacen": una etnografía sobre arreglos familiares, leonas y mujeres superpoderosas. Cuadernos de Antropología Social, 48, 55-71.

Schild, V. (2015). Emancipation as Moral Regulation: Latin American Feminisms and Neoliberalism. Hypatia, 30(3). Svampa, M. (2009). Postfacio. En Svampa, M. y Pereyra, S. Entre la ruta y el barrio. La experiencia de las organizaciones piqueteras. Buenos Aires: Biblos.

\section{Notas}

1 A lo largo del artículo, se utiliza la letra "e" en reemplazo de términos binarios en pos de incluir en los plurales no sólo los géneros masculino y femenino, sino también a las personas que no se identifican dentro de ese par.

2 En la actualidad, existen en América Latina alrededor de 1240 programas de transferencia condicionada, que operan en 18 países de la región y cubren a más de 25 millones de hogares y cerca de 113 millones de personas ( $19 \%$ de la población de la región) (CEPAL, 2010).

3 El Programa Hacemos Futuro integra a 236.767 personas (agrupando el Argentina Trabaja y el Ellas Hacen) (Ministerio de Desarrollo Social, 2019).

4 Para citar un ejemplo, el Programa Argentina Trabaja contenía en 2012 a un 54,25\% de mujeres (Ministerio de Desarrollo Social, 2015), y un año después se abriría la línea Ellas Hacen donde la población objetivo es íntegramente femenina.

5 Por ejemplo, al tiempo que el ingreso del Programa Argentina Trabaja en octubre de 2019 era de \$ 8500, el Salario Mínimo Vital y Móvil era de \$ 16875, es decir, equivale a un 50,4 \% del mismo (Resolución 6/2019 del Ministerio de Producción y Trabajo). 